\title{
VISLUMBRANDO O SIGNIFICADO DA INICIAÇÃO CIENTÍFICA A PARTIR DO GRADUANDO DE ENFERMAGEM
}

\author{
Viewing the meaning of the scientific starting from the graduating \\ nursing student
Vislumbrando el significado de la iniciación científica desde la perspectiva del estudiante de enfermería

\author{
Alacoque Lorenzini Erdmann ${ }^{1}$ \\ Keyla Cristiane do Nascimento ${ }^{3}$
}

Joséte Luzia Leite ${ }^{2}$

Gabriela Marcellino de Melo Lanzoni

\section{RESUMO}

Esta pesquisa objetivou compreender o significado da experiência de ser bolsista de iniciação científica (IC) para graduandos de enfermagem de uma universidade do sul do país. Utilizou como referencial metodológico a Grounded theory. Foram coletados dados por meio de entrevista com bolsistas e ex-bolsistas IC, totalizando dez participantes. 0 fenômeno Vislumbrando a IC como um processo de crescimento, mudança e aprendizado na graduação da enfermagem e continuidade na academia foi constituído a partir das categorias: Reconhecendo o grupo de pesquisa como espaço para educação e relacionamento interpessoal, Contrastando sentimentos em relação à atividade de pesquisa, Valorizando o aprendizado e o conhecimento adquirido em ser bolsista, Apontando o reflexo da bolsa na vida profissional e pessoale Relacionando a IC à continuidade da carreira acadêmica. Reconhecemos a importância e necessidade de incremento e valorização desta atividade na formação do graduando e do pesquisador de enfermagem e produtividade dos grupos de pesquisa.

Palavras-chave: Enfermagem. Pesquisa em Enfermagem. Atividades Científicas e Tecnológicas.

\begin{abstract}
This research aimed to understand the meaning of the experience of being scientific initiation scholarship student for nursing students from a university in the south of the country. Used as a methodological reference Grounded Theory. Data were collected through interviews with ten participants, ex-scholarship students and scholarship students. The phenomenon Viewing the scientific starting as a process of growth, change and learning in nursing graduate and continuity in academy, was formed from the categories: Recognizing the research group as a space for education and interpersonal relationships, Contrasting feelings about the activity of research, Enhancing the learning and knowledge, Showing the reflection of the scholarship in their professional and personal life and Relating to the scientific initiation scholarship with continuity of academic career. We recognize the importance and need to increase and recovery of activity in the training of students and researchers in nursing, in projects and productivity of research groups.
\end{abstract}

KEYWORDS: Nursing. Nursing research. Scientific and Technical Activities.

\section{Resumen}

El objetivo de la investigación fue comprender el significado que tiene la experiencia adquirida como becario de IC para estudiantes de enfermería de una universidad del sur del país. El marco metodológico usado fue la Grounded theory. Las entrevistas aplicadas para recolectar los datos fueron realizadas con diez becarios y ex-becarios del área de Iniciación Científica. El fenómeno Vislumbrando la IC como proceso de crecimiento, cambio y aprendizaje en el pregrado de enfermería - y su continuidad en la vida académica - se desarrolló a partir de las categorías: Identificar el grupo de investigación como espacio para la educación y las relaciones interpersonales; Contrastar los sentimientos relacionados con la actividad de investigación; Valorizar el aprendizaje y los conocimientos adquiridos durante la experiencia como becario; Resaltar la influencia que tuvo la beca en la vida profesional y personal, aplicando la IC durante la carrera académica. Queremos destacar la importancia y la necesidad que tiene desarrollar y valorizar esta actividad durante la formación del estudiante e investigador del área de enfermería y en la productividad de los grupos de investigación.

PALABRAS CLAVE: Enfermería. Investigación en enfermería. Actividades Científicas y Tecnológicas.

${ }^{1}$ Enfermeira. Professora Titular do Departamento de Enfermagem e Programa de Pós-Graduação em Enfermagem da Universidade Federal de Santa Catarina (PEN/UFSC). Doutora em Filosofia da Enfermagem. Pesquisadora do Conselho Nacional de Desenvolvimento Científico e Tecnológico (CNPq). Coordenadora da Área da Enfermagem na CAPES 2008-10. Brasil. E-mail: alacoque@newsite.com.br, ${ }^{2}$ Enfermeira. Livre Docente, Doutora Professora aposentada, Titular Emérita UNIRIO. Integrante do Núcleo de Pesquisa Gestão em Saúde e Exercício Profissional em Enfermagem (GESPEn) da EEAN/ UFRJ. Pesquisadora do CNPq. Brasil. E-mail: joluzia@gmail.com, ${ }^{3}$ Enfermeira. Doutoranda do PEN/UFSC. Membro do Grupo de Estudos e Pesquisas em Administração e Gerência do Cuidado em Enfermagem e Saúde (GEPADES). Bolsista CAPES/CNPq-Sw. Brasil. E-mail: keyla_nascimento@hotmail.com, ${ }^{4}$ Enfermeira. Mestranda do PEN/UFSC. Bolsista CNPq. Membro do GEPADES. Brasil. E-mail: gabimrc@yahoo.com.br. 


\section{INTRODUÇÃO}

A Enfermagem, enquanto ciência e profissão, tem se apropriado da pesquisa como um caminho para aprimoramento da sua atuação, sustentada pela incessante busca de novos conhecimentos. Aliada à atividade de Iniciação Científica (IC), uma modalidade de formação e incentivo à pesquisa na graduação vem incrementando a formação e o exercício profissional do enfermeiro para a competência do cuidado, dada a sua relevância e contribuições já efetivadas em diversas áreas.

A origem desta proposta vem do Conselho Nacional de Desenvolvimento Científico e Tecnológico (CNPq), que dentre as agências e institutos de pesquisa mantidos pelo governo brasileiro, se destaca por fomentar a Ciência, Tecnologia e Inovação na formação e absorção de recursos humanos e direcionar financiamentos aos projetos de pesquisa que contribuem para o aumento da produção de conhecimento e geração de novas oportunidades de crescimento para o País. ${ }^{1}$

Com as parcerias estabelecidas pelo CNPq com instituições de ensino superior e demais locais onde floresce a pesquisa, criou-se o Programa Institucional de Bolsa de Iniciação Científica (PIBIC). 0 objetivo é comum entre as duas modalidades, IC e $\mathrm{PIBIC}$, mas parte do gerenciamento desta última passa a ser de responsabilidade da instituição conveniada. ${ }^{2}$

Assim, pela concessão de bolsas de iniciação científica ao aluno de graduação, almeja-se introduzir o estudante no mundo da pesquisa científica; incentivar novos talentos potenciais entre estudantes de graduação, mediante sua participação em projetos de pesquisa orientados por pesquisador qualificado, preparandoos para o ingresso na pós-graduação; e contribuir para reduzir o tempo médio de titulação de mestres e doutores. ${ }^{1}$

A exemplo, no ano de 2007, foram concedidas à área da Enfermagem aproximadamente $12 \%$ das bolsas IC e PIBIC/ CNPq destinadas à Ciências da Saúde, ${ }^{3}$ o que corresponde a um universo de 642 bolsistas inseridos no campo da pesquisa, com a possibilidade de estabelecer maiores vínculos com a pós-graduação, voltados ao fortalecimento da Enfermagem como área de conhecimento e ao aumento da produção científica com vistas à ampliação da visibilidade e reconhecimento da profissão.

Mesmo que a atuação mais expressiva da Enfermagem brasileira em pesquisa tenha ocorrido à partir de 19704,5, 0 número de bolsas de iniciação científica cedidas à esta área ainda não é suficiente, visto que a IC deveria ser prioridade dos estudantes de graduação a fim de oportunizar a formação de um profissional com horizonte mais amplo, com valores éticos e para o desenvolvimento da capacidade de aprendizagem constantes.

Em uma perspectiva geral, sobre o impacto da iniciação científica à carreira, considera-se que a IC possibilita uma maior compreensão do mercado de trabalho por permitir a interação entre o mundo científico e as atividades práticas da profissão. ${ }^{6}$ Assim, prepara o aluno de graduação de maneira mais eficaz, desenvolvendo competências diferenciadas, principalmente quando comparado àqueles alunos que não participaram desta experiência.?

Tais benefícios oriundos da iniciação científica contribuem não somente para consolidar esta experiência como um processo que deve ser iniciado na graduação e aperfeiçoado na formação do pesquisador mestre e doutor, mas também como uma ferramenta integradora de pessoas e de momentos de produção, pois, a articulação entre os diferentes níveis de formação/ensino e a integração entre graduandos, mestrandos, doutorandos e o orientador em projetos comuns são uma oportunidade para alavancar o potencial dos alunos IC e favorecer uma visão sistêmica do trabalho cooperativo e interdisciplinar para além das fronteiras dos projetos de pesquisa. $^{8}$

Assim, visando otimizar os resultados dos investimentos na formação dos cientistas brasileiros, garantido, sobretudo, a boa utilização dos recursos públicos e o desenvolvimento da ciência nacional, há a necessidade de estudos sobre os atores da iniciação científica, para que se trace o perfil dos fatores que influenciam o alcance de seus objetivos. ${ }^{9}$

Hoje, na nossa sociedade, a competência profissional é cobrada de modo bastante ostensivo, há de se superar a busca pela titulação universitária e visar à formação de um profissional com horizonte mais amplo, com valores éticos e que tenha capacidade de aprendizagem constante.

Os ganhos do bolsista não se limitam apenas à graduação ou à pós-graduação. Aquele que opta pelo mercado de trabalho se diferencia dos demais, devido às competências desenvolvidas.

Diante dos aspectos aqui colocados, reconhecemos que a atividade de iniciação científica é importante para a formação de nossos profissionais nos diferentes níveis da academia. A partir dessas constatações, as autoras preocuparam-se com respostas aos seguintes questionamentos: Quais as atividades e/ou competências desenvolvidas na experiência de ser bolsista de iniciação científica? Quais as suas vantagens e desvantagens? Qual o significado da iniciação científica para o aluno portador desta bolsa? Com isso, busca-se compreender o significado da experiência de ser bolsista de iniciação científica para graduandos de enfermagem de uma universidade do sul do país, com vistas ao incremento e valorização desta atividade na formação do graduando, no desenvolvimento dos projetos de pesquisa e na produtividade dos grupos de pesquisa.

\section{METODOLOGIA}

0 presente estudo é fruto de um projeto de pesquisa realizado pelo Grupo de Estudos e Pesquisas em Administração e Gerência do Cuidado em Enfermagem e Saúde - GEPADES, cujo objetivo foi compreender a importância da iniciação científica para o pesquisador.

Assim, para alcançar o objetivo proposto, optamos pela Teoria Fundamentada nos Dados (TFD), também chamada 
A iniciação científica na formação do graduando Erdmann AL, Leite JL, Nascimento KC, Lanzoni GMM

Grounded Theory, como método de pesquisa. Trata-se de uma metodologia originalmente desenvolvida por sociólogos americanos, que se propuseram a elaborar uma teoria assentada nos dados a partir da exploração do fenômeno na realidade em que o mesmo se insere, sendo que a construção teórica explica a ação no contexto social. ${ }^{10}$

Pela TFD é possível acrescentar novas perspectivas e novos significados ao fenômeno, nesse caso a iniciação científica, a fim de gerar um conhecimento complexo, consolidado e fundamentado essencialmente nos dados. ${ }^{11}$

0 projeto denominado inicialmente como "A iniciação científica como formação para o pesquisador na enfermagem" teve parecer favorável do Comitê de Ética em Pesquisa em Seres Humanos da Universidade Federal de Santa Catarina, registrado sob o número 094/07. Os dados foram coletados por meio de entrevistas semiestruturadas e uma pergunta inicial: Qual o significado da iniciação científica para você? 0 encaminhamento das demais questões foi direcionado pelas pesquisadoras, a partir das respostas dos entrevistados, levando-os a refletirem sobre suas vivências e 0 desenvolvimento do seu "fazer" na experiência de ser bolsista de iniciação científica.

Optamos em realizar a pesquisa com bolsistas e ex-bolsistas de iniciação científica com experiência de mais de um ano e pertencentes ao curso de Enfermagem de uma universidade do sul do país. Desse modo, o primeiro grupo amostral foi composto por 4 ex-bolsistas e 1 bolsista de IC, que se dispuseram a integrar o grupo.

Para formar o segundo grupo amostral, composto por 2 ex-bolsistas e 3 bolsistas de IC, foram consideradas as sugestões, ideias e dúvidas que emergiram dos dados codificados e analisados a partir do primeiro grupo. A validação do Modelo Teórico propriamente dito foi efetivada com uma enfermeira ex-bolsista de IC e com uma pesquisadora expertise em Teoria Fundamentada nos Dados e que vem desenvolvendo estudos sobre a iniciação científica.

Os dados foram coletados no período compreendido entre maio e dezembro de 2007, os quais foram gravados e posteriormente transcritos, conforme prevê a metodologia adotada. Assim que se iniciou a coleta de dados, procedeu-se a análise substantiva dos dados realizada em três etapas que ocorrem de forma concomitante, a saber: codificação aberta, axial e seletiva.

Na fase de codificação aberta, os dados foram separados em partes distintas, sendo rigorosamente examinados linha à linha e comparados em busca de similaridades e de diferenças. Nesta etapa, é realizada a conceituação, ou seja, uma representação abstrata de um fato, de um objeto, de uma ação/ interação identificados como importante nos dados. ${ }^{10}$

A codificação axial é o momento de reagrupar os dados que foram divididos na codificação aberta e relacionar categorias às suas subcategorias. 0 fato de alguns códigos compartilharem características comuns ou significados permite que sejam agrupados sob conceitos mais abstratos, ou seja, subcategorias e categorias, possibilitando ao pesquisador reduzir o número de unidades com as quais trabalha. ${ }^{10}$

A última etapa é denominada codificação seletiva, ou seja, é o processo de integrar e refinar as categorias para que os resultados da pesquisa assumam a forma de teoria. É quando se torna possível desvelar a categoria central, isto é, uma categoria que expresse o tema da pesquisa, explicando sobre o que ela trata. Em seguida, chega o momento de rever o esquema teórico, na busca por consistência interna, e validar o esquema teórico. ${ }^{10}$

Para classificar e organizar conexões emergentes entre as categorias, foi utilizado um esquema organizacional, denominado por Strauss e Corbin ${ }^{10}$ como "paradigma", no qual os dados foram vislumbrados conforme condições causais, intervenientes e contextuais, estratégia e consequências.

A codificação e análise dos dados conduziram à identificação do tema central: Vislumbrando a iniciação científica como um processo de crescimento, mudança $e$ aprendizado na graduação da enfermagem $e$ continuidade na academia. A compreensão deste fenômeno foi construída por conceitos organizados em categorias que se apresentaram intimamente relacionados.

Para preservar o anonimato, as contribuições dos participantes foram identificadas, ao longo do texto, com a letra B seguida de algarismos arábicos que representam a ordem dada às entrevistas.

\section{RESULTADOS E DISCUSSÃO}

Iniciar o estudante de graduação na arte de pesquisar é 0 começo da construção do ser pesquisador, logo, se consideramos a prática também como formadora, é fundamental que 0 pesquisador em construção se reconheça como um aprendiz permanente e que, por meio do processo reflexivo, se mantenha em constante atualização para produção de conhecimento.

Assim, a iniciação científica como fenômeno de mudança e aprendizado na graduação emergiu a partir dos agrupamentos e interpretação das mensagens que os participantes expressaram durante as entrevistas, as quais formaram as seguintes categorias:

\section{Contrastando sentimentos em relação à atividade de pesquisa}

Essa categoria apresenta-se como interveniência para o alcance de melhores práticas e resultados na experiência da iniciação científica. A maior parte dos participantes expressou ter gostado de ser bolsista e se sente realizada com suas atividades, por serem remuneradas, pela flexibilidade de horário, por dispor de materiais e equipamentos para realização da pesquisa, bem como por ter acompanhado todas as etapas dos projetos, inclusive a divulgação dos resultados. Outros 
A iniciação científica na formação do graduando Erdmann AL, Leite JL, Nascimento KC, Lanzoni GMM

apontaram que sua vivência como bolsista foi permeada por momentos bons e ruins, pois a demanda de atividades da pesquisa ocupou todo seu tempo livre, dificultando a realização de estágios práticos extracurriculares e a conciliação da pesquisa com suas outras atividades e compromissos. Ainda, perceberamse realizando atividades consideradas "tarefeiras" e/ou que vão além da pesquisa e, por esse motivo, desassociaram o ser bolsista da carreira acadêmica.

Acho que para o que a gente se propôs a fazer, a gente conseguiu, e por isso acredito que meu trabalho colaborou e me sinto realizada. (B07)

Eu me sentia muito feliz e realizada executando as tarefas mais simples para sustentar as mais complexas que viriam posteriormente. (B08)

0 bolsista muitas vezes é reconhecido por muitos como "secretário" e isso o torna um pouco tarefeiro. (B02)

0 desvio do estudante para a execução de atividades burocráticas, reduzindo-o a mão-de-obra barata dentro das instituições de ensino, é uma das imprecisões do programa. ${ }^{12}$ Certamente, todos perdem nesta situação: o orientador, que não fomentou momentos de troca com o estudante, desperdiçando contribuições diferentes e muitas vezes inovadoras, o bolsista, que não teve oportunidade de explorar, de forma supervisionada, o mundo da pesquisa e, principalmente, a sociedade, que deixa de ampliar seu desenvolvimento social e econômico.

Para Reis ${ }^{6}$ houve contraste no quesito satisfação em relação ao mérito científico do projeto realizado durante o período vigente da bolsa. Nesta mesma pesquisa também reconhece na bolsa um auxílio valioso para os estudos dos jovens. Este auxílio financeiro é destinado à aquisição de materiais necessários para sua formação, bem como a ajudar no orçamento familiar, exercendo, desta forma, uma responsabilidade diferente daquela exclusivamente científica: a social. ${ }^{12}$

\section{Reconhecendo o grupo de pesquisa como espaço para educação e relacionamento interpessoal}

Esta categoria é considerada o contexto e as condições causais nos quais o fenômeno Vislumbrando a iniciação científica como um processo de crescimento, mudança e aprendizado na graduação da enfermagem e continuidade na academia ocorre, reconhecendo o grupo de pesquisa como 0 espaço no qual se desenvolvem as ações e interações entre o bolsista e os demais membros do grupo de pesquisa.

Os grupos de pesquisa destacam-se como cenário de formação de futuros pesquisadores por meio da realização de reuniões visando acolher novos membros e integrá-los da dinâmica de trabalho. Com destaque, a articulação entre bolsistas, mestrandos, doutorandos e orientador no desenvolvimento dos projetos foi citada como principal motivação para o aprendizado do bolsista e facilitador na sua inserção e evolução enquanto pesquisador.

Acho que o Grupo te ensina a pesquisar, isso se torna uma prática comum e necessária. (B06) 0 trabalho em grupo me permitiu observar o que as pessoas têm de melhor, o que sabem fazer melhor e ir a busca deste conhecimento, principalmente quando elas têm disponibilidade para ensinar. Então, também despertei para ensinar o que eu sabia de melhor para elas, fazendo do processo educativo algo construtivo. (B08)

0 Grupo de Pesquisa dispõe de um espaço importante para a troca de saberes, é um local com estrutura física adequada, material, computadores, e o mais importante: as pessoas. (B03)

A partir do convívio com pesquisadores mais experientes e o vínculo com um grupo de pesquisa competente, é possível afirmar que o bolsista consegue entender de maneira precoce sobre ciência atualizada, podendo contribuir enormemente com ideias criativas e sensatas, sendo estas constatadas em muitos trabalhos científicos dos estudantes de pós-graduação provenientes da iniciação científica. ${ }^{12}$

Assim, o exercício da iniciação científica deve transcorrer em ambiente de investigação que integra pessoas, espaços e momentos de produção. ${ }^{2}$ Com o crescimento significativo dos grupos de pesquisa da área da Enfermagem no contexto nacional, tornou-se oportuno o aumento das publicações e qualificação dos integrantes, que são constituídos por estudantes, pesquisadores e pessoal de apoio técnico, ${ }^{5}$ tornando efetiva a rica articulação entre os diversos níveis de formação e, mesmo, campos da ciência para a construção do conhecimento alicerçado em diferentes olhares.

\section{Valorizando o aprendizado e o conhecimento adquirido em ser bolsista}

A iniciação científica pode ser compreendida como uma oportunidade de ilimitadas aprendizagens pelo acadêmico que está em formação, sendo uma experiência que nasce por uma inquietação, na busca pelo conhecimento, da curiosidade e do desejo de conhecer assuntos até então não emergentes. ${ }^{13}$

Assim, o tema central é desencadeado pelo interesse do aluno de graduação em pesquisar, valorizando o aprendizado e o conhecimento que pode vir a adquirir em consequência de ser bolsista. Compreende, ainda, que durante a iniciação científica se aprofundaram saberes que não iriam desenvolver na graduação, relacionando a experiência como bolsista à de apreender o máximo de informação e ampliar conhecimento. Acho que no grupo a gente desenvolve assuntos que, se eu não participasse, não iria aprofundare pesquisar tanto. (B06) 
A iniciação científica na formação do graduando

Erdmann AL, Leite JL, Nascimento KC, Lanzoni GMM

Ser bolsista é estar em contato com novos conhecimentos e aberta a mudanças de pensamentos. (B10)

Posso afirmar que, no início, nosso papel é mais de esponja, absorvendo o máximo de informação possível. (B08)

A analogia apresentada por um dos participantes, de 0 bolsista ser uma "esponja", reflete a compreensão de que a experiência da iniciação científica é um momento de grande imersão no conhecimento científico, em conteúdos e perspectivas que não seriam abordadas em outros espaços da universidade ou de sua vida cotidiana.

Assim, perder o medo do novo é considerado uma vantagem alcançada pelos bolsistas, pois o processo de aprendizado apoiado na diretriz de um orientador traz uma certa habilidade e facilidade para interpretar uma determinada situação e reconhecer se énecessário buscar mais informações para resolvêla. ${ }^{12}$ Há um desvelamento crítico sobre a realidade apresentada, que torna a busca por mais informações uma aventura prazerosa e que se retroalimenta.

Apontando o reflexo da bolsa na sua vida profissional e pessoal e Relacionando a bolsa IC à continuidade da carreira acadêmica

Essas categorias serão analisadas em conjunto, uma vez que os participantes evidenciaram as repercussões da Iniciação Científica na sua carreira acadêmica, tanto como estratégia quanto como consequência.

A utilização de técnicas e métodos científicos durante 0 curso de graduação oferece ao estudante a vivência plena da vida acadêmica, que interfere positivamente no posterior exercício profissional. ${ }^{14}$ Desta forma, os participantes evidenciam o amadurecimento e a autonomia como características proporcionadas pela bolsa IC, ressaltando 0 respeito às opiniões, perceber o que as pessoas tem de melhor, a necessidade permanente de atualização, ter força de vontade e superar desafios, ser crítico e estar aber to para o leque de novos conhecimentos e caminhos que a pesquisa revela.

Logo, o acadêmico integrado a um projeto de pesquisa científica tem um maior contato com a prática, além de maior oportunidade de aplicabilidade de seus conhecimentos universitários. Ainda, os conteúdos desenvolvidos durante a experiência da bolsa contribuiu para a formação profissional dos estudantes aproximando-os dos desafios do mundo do trabalho. ${ }^{6}$

A aplicação repetitiva do pensamento lógico e crítico, atrelada à articulação do pensamento científico e teórico para a práxis, são fatores ímpares para a formação adequada de novos pesquisadores e profissionais qualificados. ${ }^{6}$

No que se refere ao despertar para a carreira acadêmica, no grupo estudado, a IC cumpriu a sua função, pois a maioria
Esc Anna Nery Rev Enferm 2010 jan-mar; 14 (1): 26-32

tem desejo de cursar ou já está cursando o mestrado. Apenas duas bolsistas relataram não ter interesse em iniciar imediatamente outro curso, pois pretendem primeiro atuar na assistência. No entanto, ressaltaram que irão manter o vínculo com os grupos de pesquisa paralelamente à atuação profissional e mais tarde ingressar na pós-graduação.

Embora, a iniciação científica vise à formação de mestres e doutores em tempo reduzido, quando o bolsista faz a opção pelo exercício profissional em um campo diferente do acadêmico também usufrui das habilidades desenvolvidas, como: melhor capacidade de análise crítica, maturidade intelectual e, seguramente, maior discernimento para enfrentar as suas dificuldades. $^{12}$

No entanto, a repercussão das bolsas de iniciação científica tem sido positiva para a diminuição do tempo de ingresso do mestrado e do doutorado, sendo que os ingressantes são mais jovens e tendem a ter uma continuidade na sua formação. Quanto à interferência da iniciação científica no tempo de formação de pesquisadores, os mestrandos que tiveram contato com a IC despendem em média dois anos e meio para entrar no mestrado, enquanto os não bolsistas levaram em média sete anos. Já no doutorado, os alunos que tiveram bolsa de IC despendem em média sete anos para ingressar, enquanto os que não tiveram acesso a bolsas de IC tardaram em média 11 anos. ${ }^{15}$

Eu acho que se tu te propões a participar de grupo de pesquisa, e se tu não utilizar isso depois para eu mestrado ou um doutorado, acho que é um trabalho jogado fora, acho que é um desperdício. (B09)

Minha bolsa para mim, desde o início, foi um trampolim para o mestrado. (B05)

Minha participação no grupo de pesquisa possibilitou lapidar duas grandes áreas, ou seja, pessoal e profissional. (B02)

Corroborando os achados, Medeiros ${ }^{7}$ confirma a interferência da iniciação científica na vida pessoal e profissional dos estudantes e atribui a ela o mérito de determinar a escolha pelo mestrado, bem como pela área profissional acadêmica. Ainda, deve-se destacar o diferencial a favor do programa e da repercussão da iniciação científica no currículo dos estudantes, pois, em alguns concursos para docentes, a disputa pelas vagas ficaram, quase que exclusivamente, com os ex-bolsistas, pois os demais não se sentiam tão preparados para competir. ${ }^{12}$ Isso fortalece, um dos objetivos do programa de iniciação científica: acesso rápido ao programa de pós-graduação com titulação em prazo adequado visando à formação de jovens pesquisadores. ${ }^{5}$

A organização das inter-relações entre as categorias encontradas apontaram à conformação do fenômeno Vislumbrando a iniciação científica como um processo de crescimento, mudança e aprendizado na graduação da enfermagem e continuidade na academia, representado pela Figura 1. 
Figura 1: Apresentando o fenômeno Vislumbrando a iniciação científica como um processo de crescimento, mudança e aprendizado na graduação da enfermagem e continuidade na academia.

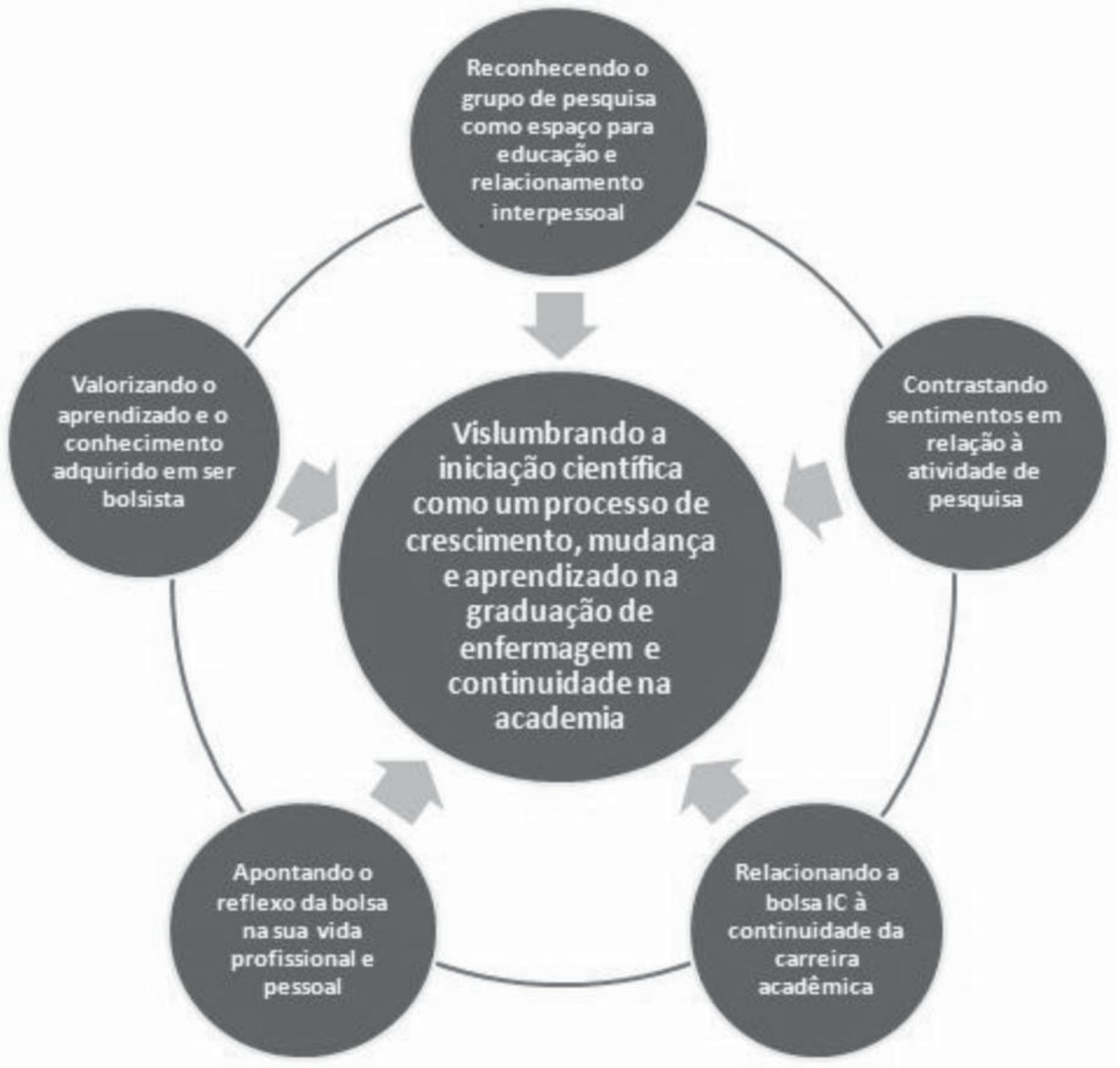

Este processo de crescimento, mudança e aprendizado se instala quando se Reconhece o grupo de pesquisa como espaço para educação e relacionamento interpessoal (contexto e condições causais), cujas atividades realizadas permitem Contrastar sentimentos em relação à atividade de pesquisa (condição interveniente) e Valorizar o aprendizado e o

\section{CONSIDERACÕES FINAIS}

A compreensão do significado da experiência de ser bolsista de iniciação científica para graduandos de enfermagem de uma universidade do sul do país possibilitou desvelar o fenômeno Vislumbrando a iniciação científica como um processo de crescimento, mudança e aprendizado na graduação da enfermagem e continuidade na academia.

Reconhecemos a importância e a necessidade de incremento e valorização desta atividade na formação do graduando de enfermagem, no desenvolvimento dos projetos de pesquisa e conhecimento adquirido em ser bolsista (consequência). Na experiência deste processo, reconhece-se a sua importância e aponta-se o Reflexo da bolsa na vida profissional e pessoale Relaciona-se a bolsa IC à continuidade da carreira acadêmica (estratégia e consequência).

na produtividade dos grupos de pesquisa, de modo que a participação dos graduandos nessas atividades deva ser cada vez mais incentivada.

Além de configurar importante etapa na aprendizagem, os graduandos IC recebem a indiscutível vantagem de formação para o fazer/pesquisar, pelo acompanhamento/ desenvolvimento/participação em projetos de pesquisas e experiências acadêmicas oportunizadas por seus orientadores.

Por meio das atividades de pesquisa proporcionadas pelo programa de IC, o bolsista desperta para o questionamento 
A iniciação científica na formação do graduando

Erdmann AL, Leite JL, Nascimento KC, Lanzoni GMM

crítico e o desenvolvimento de habilidades instrumentais para aplicação de métodos científicos. A realização de suas investigações iniciais e divulgação destas em eventos científicos bem como a relação estreita criada entre orientador e orientando são formas de construir uma atitude positiva e uma autoconfiança nesses alunos que têm o privilégio de poderem desfrutar desse contexto no decorrer da graduação.

Este estudo apresenta as limitações de uma pesquisa qualitativa, a qual não visa generalizações; no entanto, seus

\section{REFERÊNCIAS}

1.Conselho Nacional de Desenvolvimento Científico e Tecnológico- CNPq O CNPq. [citado 24 nov 2008] Disponível em:<http://www.cnpq.br/ cnpq/index.htm>

2.Erdmann AL, Pagliuca LMF. Iniciação científica: trajetória para a pesquisa. OBJN 2007; 6(0). [citado 3 fev 2009] Disponível em: <http:/ /www.uff.br/objnursing/index.php/nursing/article/view/767/173>

3.Conselho Nacional de Desenvolvimento Científico e Tecnológico-CNPq. Investimentos do CNPq em CT\&l [citado 3 jan 2009] Disponível em: $<$ http://www.cnpq.br/estatisticas/index.htm>

4.Erdmann AL, Mendes IAC, Leite JL. A enfermagem como área de conhecimento no CNPq: resgate histórico da representação de área. Esc Anna Nery Rev Enferm 2007 mar; 11(1): 118-26.

5.Erdmann AL, Lanzoni GMM. Características dos grupos de pesquisa da enfermagem brasileira certificados pelo CNPq de 2005 a 2007. Esc Anna Nery Rev Enferm 2008 jun; 12 (2): 316 - 22.

6. Reis LA. Programa Institucional de Bolsas de Iniciação Científica do IBAMA: uma política de pesquisa [dissertação de mestrado]. Brasilia(DF): Programa de Pós-Graduação em Educação/ UCB; 2007.

7.Medeiros RASM. 0 impacto do Programa de Iniciação Cientíica (CNPq) na carreira do graduando, à luz dos fenômenos de mentoria e de competência: o caso dos alunos do curso de administração da UFPE [dissertação de mestrado]. Recife(PE): Programa de PósGraduação em Administração/ UFPE; 2005.

8.Radünz V, Erdmann AL. Organização de um sistema de compartilhamento e intercâmbio de produção de conhecimento entre
Esc Anna Nery Rev Enferm 2010 jan-mar; 14 (1): 26-32

achados podem ser aplicados com grupos de pessoas e em realidades com características semelhantes. Conscientes do papel formativo da iniciação científica, como complemento dos subsídios oferecidos aos discentes em salas de aula e como preparo de profissionais para a pós-graduação, esperamos a permanência e ampliação de propostas similares a ela para o fortalecimento e aumento da visibilidade da Enfermagem como uma profissão produtora de conhecimento.

a enfermagem da região sul do Brasil. Texto\&Contexto Enferm 1996; 5(2): 258-70.

9.Görgens JB. Avaliação da produção científica dos egressos, bolsistas e não bolsistas de iniciação científica, do curso de medicina da Universidade Federal de Minas Gerais, de 1994 a 1999, pelo Currículo Lattes [tese de doutorado]. Belo Horizonte(MG): Faculdade de Medicina. Programa de Pós-Graduação em Saúde da Mulher/UFMG; 2007.

10.Strauss A, Corbin J. Basics of qualitative research: grounded theory procedures and techniques. Thousand Oaks, CA: Sage; 1990.

11.Betinelli LA. A solidariedade no cuidado: dimensão e sentido da vida [tese de mestrado]. Florianópolis(SC): Programa de PósGraduação em Enfermagem/ UFSC; 2002.

12.Fava-de-Moraes F, Fava M. A iniciação científica: muitas vantagens e poucos riscos. Perspect 2000 jan; 14(1): 73-7.

13.Kirsch DB. A iniciação científica na formação inicial de professores: repercussões no processo formativo de egressas do curso de pedagogia [dissertação de mestrado]. Santa Maria(RS): Programa de Pós-Graduação em Educação/UFRGS; 2007. 111 f.

14. Rios MI. Iniciação científica: uma verificação de seus objetivos na UFMG [monografia]. Belo Horizonte(MG): Faculdade de Ciências Econômicas/UFMG; 2000.

15. Velloso J, Velho L. Mestrandos e doutorandos no país: trajetórias de formação. Brasília(DF): Capes / Unesco; 2001. 